\title{
DESEMPENHO DO COMPÓSITO DE MATRIZ POLIÉSTER REFORÇADO COM TECIDO DE JUTA EM UM SISTEMA DE BLINDAGEM MULTICAMADA*
}

\section{Resumo}

\author{
Foluke Salgado de Assis ${ }^{1}$ \\ Sérgio Neves Monteiro ${ }^{1}$ \\ Fabio Da Costa Garcia Filho' \\ Artur Camposo Pereira ${ }^{1}$ \\ Édio Pereira Júnior ${ }^{1}$ \\ Carlos Luiz Ferreira ${ }^{1}$
}

O desempenho balístico de compósitos de matriz de poliéster reforçados com tecido de juta foi avaliado como segunda camada em um sistema de blindagem multicamadas (MAS - Multilayered Armor Systems). Diferentes frações volumétricas de tecido de juta, até $30 \%$ vol., foram misturadas com poliéster ortoftálica para se fabricar as placas compósitas. Estes compósitos foram ligados a uma camada cerâmica de alumina dianteira e seguidos por uma placa metálica de liga de alumínio como terceira camada do MAS, os testes balísticos foram conduzidos usando munição comercial de alta velocidade de $7,62 \mathrm{~mm}$. A profundidade de penetração causada pela bala em um bloco de plastilina, que simula o corpo humano, foi usada para avaliar o desempenho balístico do MAS de acordo com o padrão internacional. Os materiais fraturados após testes foram analisados por microscopia eletrônica de varredura (MEV). Os resultados indicaram que os compósitos de tecido de juta apresentam um desempenho semelhante ao do tecido de aramida comercialmente utilizado como segunda camada para MAS com mesma espessura, o Kevlar ${ }^{\mathrm{TM}}$. O mecanismo que explica este comportamento balístico semelhante, bem como as vantagens econômicas comparativas dos compósitos de tecido de juta foram discutidos.

Palavras-chave: Tecido de juta; Compósito de poliéster; Comportamento balístico; Sistema de blindagem multicamada.

\section{PERFORMANCE OF JUTE FABRIC REINFORCED POLYESTER MATRIX COMPOSITE IN MULTILAYERED BALLISTIC}

\section{Abstract}

The ballistic performance of jute fabric-reinforced polyester matrix composites was evaluated as second layer in a multilayered armor system (MAS) with front aluminabased ceramic tile. Different volume fractions of jute fabric, up to $30 \mathrm{vol} \%$ were mixed with orthophthalic polyester to fabricate laminate composites. These composites were bonded to the front ceramic and followed by an aluminum alloy as third MAS layer, Ballistic tests were conducted using commercial high velocity $7.62 \mathrm{~mm}$ ammunition. The depth of penetration caused by the bullet in a block of clay witness, simulation a human body was used to evaluate the MAS ballistic performance according to international standard. The fractured materials after tests were analysed by scanning electron microscopy (SEM). The results indicated that jute fabric composites present a performance similar to that of the much stronger aramid fabric laminate, the commercial $\operatorname{Kevlar}^{\mathrm{TM}}$, as conventional MAS second layer with same thickness. The mechanism which explains this similar ballistic behavior as well as the comparative economical advantages of the jute fabric composites were discussed.

Keywords: Jute fabric; polyester composite; multilayered armor; ballistic performance. 


\section{INTRODUÇÃO}

A proteção balística de pessoal, equipamentos e veículos se torna cada vez mais importante devido a conflitos armados urbanos e guerras regionais que envolvem cada vez mais poder de fogo. Em particular, o uso de munições de alta velocidade, impacto e potência, como a de classe III 7,62 x $51 \mathrm{~mm}$ [1], constituem uma grande ameaça pessoal, sejam policiais, soldados ou civis. Neste caso, as coletes de blindagem de camada única, como as feitas apenas em aço ou Kevlar ${ }^{\mathrm{TM}}$, exigiriam uma espessura muito grande e, consequentemente, dificultariam a mobilidade do usuário. Para proteção contra munições de alta velocidade, o sistema de blindagem multicamadas (MAS) composto por um material frontal rígido e quebradiço se apresenta como uma solução efetiva [2-5]. O MAS típico é composto de um camada frontal de polietileno de ultra alto peso molecular (UHMWPE) com o objetivo de absorver a maior parte da energia de impacto de bala por fragmentação em partículas finas. Uma segunda camada que absorverá a energia de impacto restante associada à nuvem de fragmentos supersônicos gerados no impacto frontal, tradicionalmente laminados de tecido de aramida tais como Kevlar $^{\mathrm{TM}}$ e Twaron $^{\mathrm{TM}}$ $[2,6]$, bem como tecidos de UHMWPE, como Dyneema ${ }^{\mathrm{TM}}$ e Spectra $^{\mathrm{TM}}$ [7-8]. A terceira camada do sistema de blindagem, normalmente uma folha metálica dúctil pode ser adicionada para reduzir ainda mais a energia transportada pela onda de choque de impacto [9]. Em um teste balístico, um bloco de plastilina simula um corpo humano para ser protegido pelo MAS, e só deve permitir a penetração dos fragmentos transportados pela onda de choque de impacto até um limite padrão de $44 \mathrm{~mm}$ [1]. Acima desta profundidade de indentação no bloco de plastilina, o teste balístico indicaria um trauma letal para o corpo humano.

A eficiência da segunda camada do MAS envolve uma interação complexa com a onda de choque e fragmentos provenientes do impacto inicial da bala. De fato, a onda de choque gerada por impacto se propagará dentro do MAS e será parcialmente refletida em cada interface [3,10]. A densidade dos materiais do sistema afeta a eficiência da absorção de energia. O comportamento dinâmico de um sistema de blindagem multicamada sujeito ao impacto de um projetil é amplamente estudado [10,11] e justifica a escolha de um material leve como segunda camada no MAS.

Em geral, a utilização do Kevlar ${ }^{\mathrm{TM}}$ é, hoje, a opção mais comum. No entanto, materiais naturais e mais baratos poderiam ser usados como segunda camada de um MAS. Por exemplo, compósitos reforçados com fibras lignocelulósicas naturais vêm sendo amplamente investigados como alternativas econômicas e sustentáveis para substituir os compósitos de fibras sintéticas [12-19]. As aplicações bem sucedidas de compósitos de fibras naturais são relatadas na indústria automotiva [20-22], mas recentemente esses compósitos estão sendo investigados em blindagens balísticas [23-31]. Tanto compósitos reforçados com fibras naturais, como com tecidos apresentaram desempenho balístico comparável como Kevlar ${ }^{\mathrm{TM}}$, quando usado na segunda camada do MAS [26-31]. Em particular, em um trabalho anterior, um compósito de epóxi reforçado com 30 vol. \% de tecido de juta, empregado como segunda camada de um MAS com cerâmica dianteira, parou com sucesso a perfuração de uma bala de 7,62 mm [30]. A profundidade de indentação produzida atendia ao padrão e era igual à associada ao MAS com Kevlar ${ }^{\text {TM }}$ como segunda camada. No entanto, a integridade do compósito de tecido de juta não foi investigada nem a impedância da onda de choque do sistema. 
No presente trabalho, o desempenho balístico de um MAS semelhante ilustrado esquematicamente na Fig. 1 foi estudado, usando o compósito de poliéster reforçado com 10, 20 e $30 \%$ vol de tecido de juta. A integridade dos compósitos e a impedâncias das ondas de choque foram avaliadas.

\section{MATERIAIS E MÉTODOS}

Os sistemas de blindagem multicamadas (MAS), como o mostrado esquematicamente na Fig. 1, foram testes balisticamente de acordo com a norma NIJ. [1]

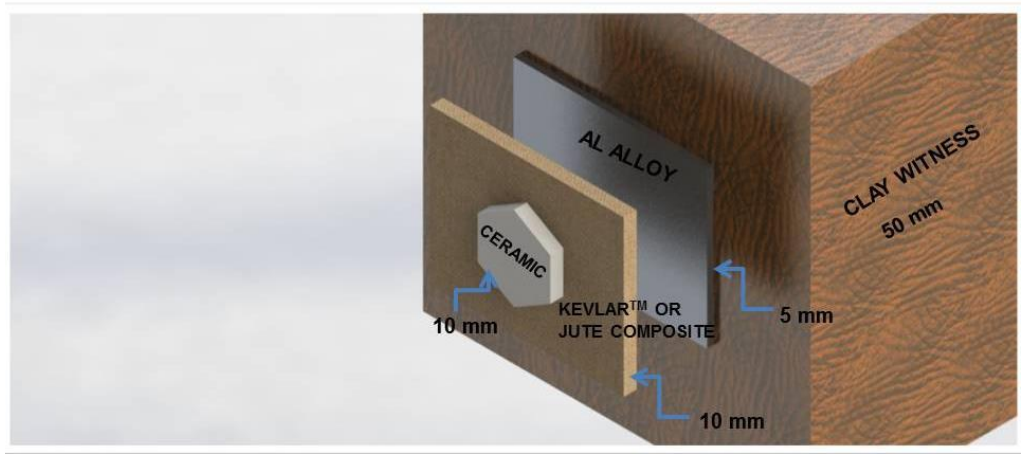

Figura 1. Representação esquemática de um sistema de blindagem multicamada a frente do bloco de plastilina

Cada sistema foi composto de uma placa frontal de cerâmica hexagonal $\mathrm{Al}_{2} \mathrm{O}_{3}-4 \%$ em peso de $\mathrm{Nb}_{2} \mathrm{O}_{5}$. Tais cerâmicas foram sinterizadas a $1400^{\circ} \mathrm{C}$ por 3 horas, a partir de uma mistura de pó puro de $\mathrm{Al}_{2} \mathrm{O}_{3}$, fornecido pela Treibacher Scheifmittel e do pó de $\mathrm{Nb}_{2} \mathrm{O}_{5}$ fornecido pela Companhia Brasileira de Mineração e Metalurgia. $\mathrm{O}$ tamanho final de grão cerâmico foi de cerca de $4 \mu \mathrm{m}$.

Colado na cerâmica frontal, uma segunda camada de placa compósita de dimensões $150 \times 120 \times 10 \mathrm{~mm}$ foi produzida intercalando camadas de tecido de juta, fornecido pela firma Lealtex, com poliéster ortoftálico com $1 \%$ em peso de metilcetona, como endurecedor, ambos fornecidos pela empresa Resinpoxy. Depois de colocar em um molde de aço a quantidade necessária de tecido e poliéster para uma placa final de $10 \mathrm{~mm}$ de espessura, uma pressão de $3 \mathrm{MPa}$ foi aplicada por $24 \mathrm{~h}$. Por fim, uma terceira camada de dimensões $150 \times 120 \times 5 \mathrm{~mm}$ de liga de aluminio 5052 H34 foi utilizada para completar o MAS. A colagem do sistema foi feita com cola comercial Sikaflex ${ }^{\mathrm{TM}}$ da Sika Co.

Os ensaios balísticos, ilustrados esquematicamente na Fig. 2, foram realizados no Centro de Avaliação do Exército (CAEx) e foram conduzidos de acordo com a norma, utilizando munição de classe III 7,62 x $51 \mathrm{~mm}$ localizado a $15 \mathrm{~m}$ do alvo. A velocidade da bala de 7,62 $\mathrm{mm}$ (chumbo com 9,7 g) foi medida por barreiras ópticas e radar Doppler. 


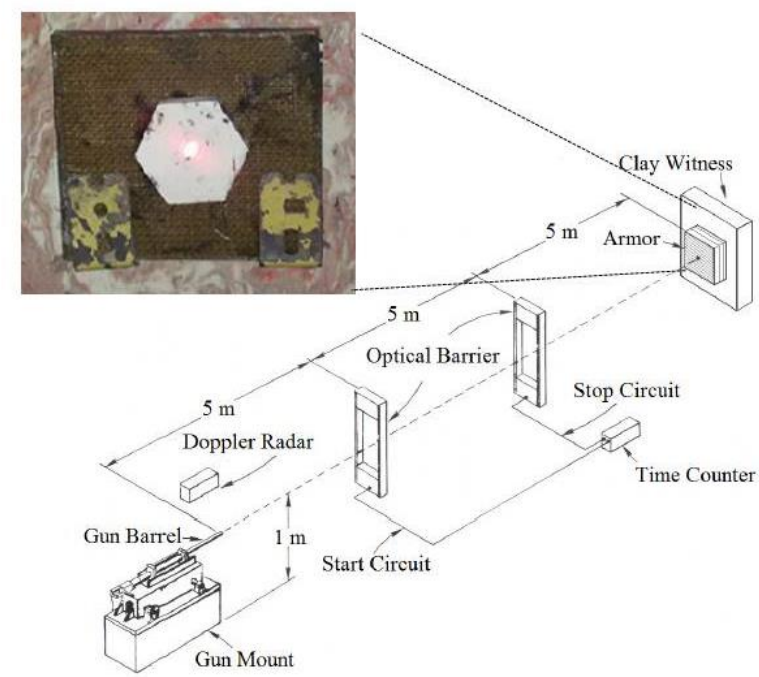

Figura 2. Desenho esquemático do túnel de tiro do CAEx e imagem real de MAS antes de ser ensaiado

Após cada teste balístico, que não tenha perfurado o sistema de blindagem, uma indentação foi produzido no bloco de plastilina, tais medidas foram realizadas com um sensor laser com 0,01 mm de precisão, mostrado na Fig. 3. A estatística de Weibull foi utilizada para analisar a profundidade dos resultados de indentação.

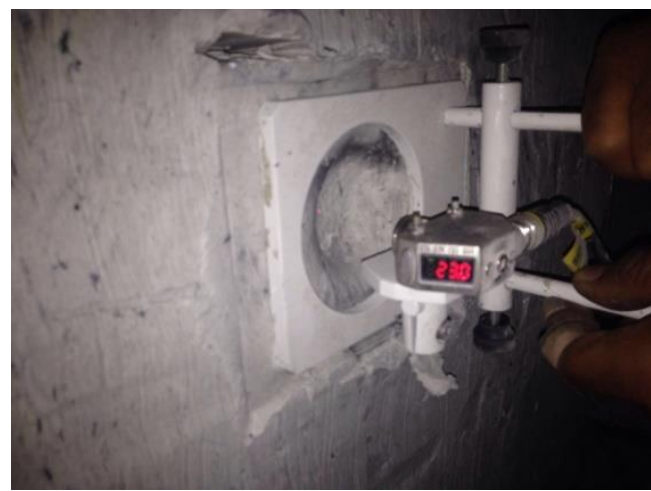

Figura 3. Profundidade de endentação sendo medida com sensor laser

As analises de fratura após o ensaio balístico foram realizadas através de microscopia eletrônica de varredura (MEV). A densidade dos componentes foi determinada pelo método de Arquimedes.

\section{RESULTADOS E DISCUSSÃO}

Todos os testes balísticos realizados neste trabalho a energia de impacto não foi capaz de perfurar a camada metálica do MAS. Esta camada foi deformada plasticamente e causou uma profundidade de indentação no bloco de plastilina, como mostrado na Figura 3, menor que $44 \mathrm{~mm}$, o limite exigido pelo padrão NIJ. A Figura 4 ilustra o aspecto dos diferentes alvos MAS após os testes balísticos. Notase que a cerâmica hexagonal frontal desapareceu por completo fragmentando em todos os alvos. Além disso, no MAS da Fig. 4a, sua segunda camada de $10 \%$ em volume de compósito de tecido de juta também desapareceu por desintegração completa. Quanto ao MAS na Fig. 4b, sua segunda camada de $20 \%$ em volume de composto de tecido de juta foi parcialmente desintegrada. A única segunda camada, 
a Fig. 4c, que permaneceu intacta, foi a de $30 \%$ em volume de compósito de tecido de juta.
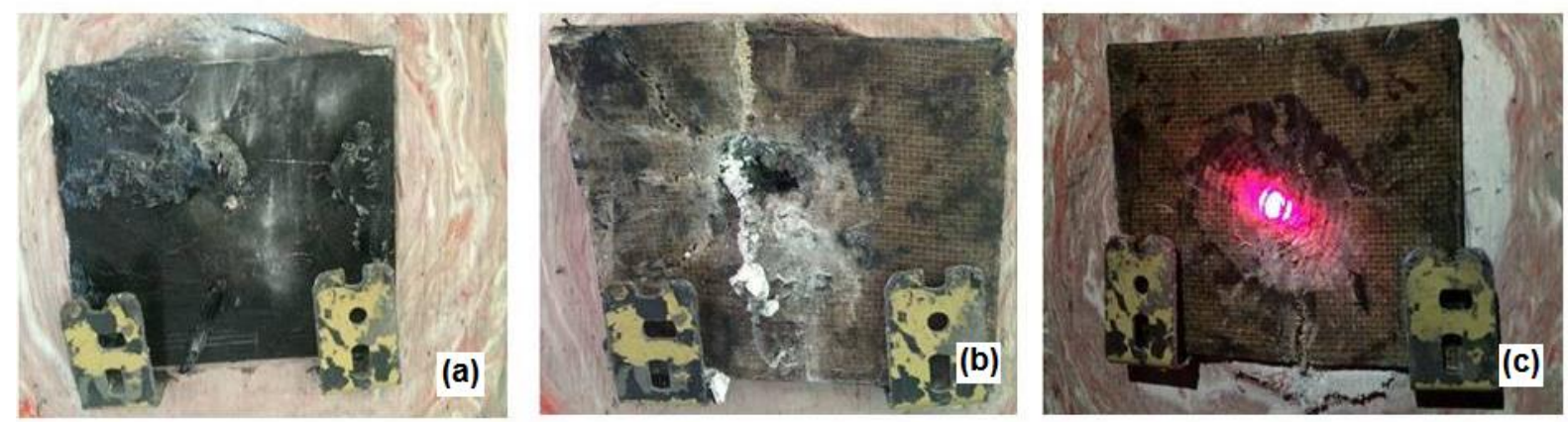

Figura 4. Aspecto dos MAS após o ensaio balístico (a) $10 \%$, (b) $20 \%$ e (c) $30 \%$ de volume de tecido de juta

A Tabela 1 apresenta os valores médios e os parâmetros de Weibull correspondentes para a medida da Figura 4, além dos resultados obtidos para 0 Kevlar $^{\text {TM }}$ que foi medido em outros trabalhos [26-31].

Tabela 1. Profundidade de endentação e parâmetros de Weibull

\begin{tabular}{cccc}
\hline Segunda camada do MAS & $\begin{array}{c}\text { Profundidade de } \\
\text { indentação }(\mathrm{mm})\end{array}$ & Módulo $(\beta)$ & Precisão $\left(\mathrm{R}^{2}\right)$ \\
\hline Poliéster com 10\%vol tecido de juta & $18 \pm 3$ & 6.81 & 0.96 \\
Poliéster com 20\%vol tecido de juta & $23 \pm 3$ & 9.29 & 0.94 \\
Poliéster com 30\%vol tecido de juta & $17 \pm 2$ & 9.76 & 0.98 \\
KevlarTM $^{\text {TM }}$ & $21 \pm 3$ & 8.43 & 0.90 \\
\hline
\end{tabular}

Os resultados da profundidade de indentação na Tabela 1 são, dentro do erro, praticamente iguais. Embora surpreendentes, resultados similares também tenham sido encontrados para outras fibras naturais / tecido [26-31]. O motivo desse desempenho balístico similar é a capacidade da segunda camada, com cerâmica frontal, para coletar fragmentos gerados a partir do impacto balístico [32]. Esta habilidade não requer fibras mais fortes, mas mecanismos de incrustação mecânica, atração de fragmentos pelas forças de Van der Waals e cargas estáticas na superfície da fibra sintética ou natural. A Figura 5 mostra as fractográfias obtidas por MEV que ilustram o mecanismo de captura de fragmentos (partículas brancas) pela segunda camada do compósito reforçado com tecido de juta de $30 \%$ em volume.
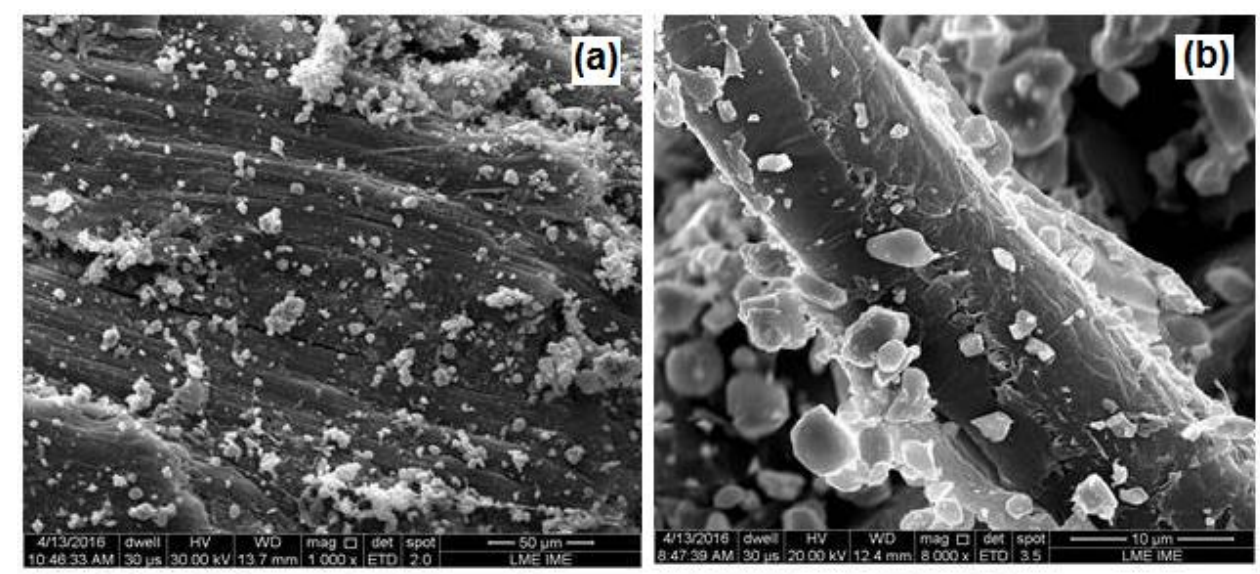

Figura 5. Análise fractográfica por MEV do compósito com $30 \%$ de volume de tecido de juta 
A Tabela 2 apresenta os resultados da análise de correspondência de impedância. Em princípio, seria de esperar que quanto menor for a impedância de choque da segunda camada, menor será a profundidade de indentação no bloco de plastilina.

Tabela 2. Resultados da análise de impedância

\begin{tabular}{ccccc}
\hline $\begin{array}{c}\text { Segunda camada do } \\
\text { MAS }\end{array}$ & $\begin{array}{c}\text { Up } \\
(\mathrm{m} / \mathrm{s})\end{array}$ & $\begin{array}{c}\mathrm{P} \\
(\mathrm{GPa})\end{array}$ & $\begin{array}{c}\mathrm{Us} \\
(\mathrm{m} / \mathrm{s})\end{array}$ & $\begin{array}{c}\mathrm{Z} \\
\left(10^{6} \mathrm{~kg} / \mathrm{m}^{2} . \mathbf{s}\right)\end{array}$ \\
\hline $\begin{array}{c}\text { Poliéster com 30\%vol } \\
\text { tecido de juta }\end{array}$ & 724 & 2.61 & 3,105 & 3.60 \\
$\begin{array}{c}\text { Poliéster com 20\%vol } \\
\text { tecido de juta }\end{array}$ & 733 & 2.34 & 2,798 & 3.19 \\
$\begin{array}{c}\text { Poliéster com 10\%vol } \\
\text { tecido de juta }\end{array}$ & 741 & 2.02 & 2,440 & 2.74 \\
$\quad$ Kevlar $^{\mathrm{TM}}$ & 715 & 2.99 & 2,909 & 4.19 \\
\hline
\end{tabular}

É importante notar que todos os compósitos de tecido de juta como segunda camada possuem valores significativamente menores do que Kevlar ${ }^{\mathrm{TM}}$. Entre eles, os compósitos de tecido de juta exibem diferenças relativamente pequenas no valor de Z. Portanto, pode-se inferir que a proteção balística não deve ser muito diferente. As impedâncias de ondas de choque definidas pela Eq.1 foram calculadas usando a análise correspondente para todos os diferentes tipos de MAS com compósitos de tecido de juta como segunda camada.

$$
Z=\rho U_{s}
$$

Equação 1

Onde $\rho$ é a densidade do material e Us é a velocidade da onda de choque no material.

Com o objetivo de avaliação, a dissipação de energia de impacto por cada compósito de tecido de juta sozinho, isto é, separada do sistema multicamada. Nestes testes, as velocidades da bala de $7,62 \mathrm{~mm}$, antes e depois da perfuração da placa compósita foram medidas. Essa velocidade de impacto $\left(\mathrm{V}_{\mathrm{i}}\right)$ e velocidade residual $\left(\mathrm{V}_{\mathrm{r}}\right)$ permite o cálculo da energia dissipada $(\Delta \mathrm{Ed})$ no interior do compósito.

$$
\Delta E_{d}=\frac{1}{2} m\left(v_{i}^{2}-v_{r}^{2}\right)
$$

Equação 2

Onde $\mathrm{m}=9.7 \mathrm{~g}$ é a massa de bala de chumbo.

A Tabela 3 apresenta o impacto e as velocidades residuais, bem como a energia dissipada internamente, a partir de testes balísticos de compósitos de tecido de juta individuais. Nesta tabela, também são apresentados resultados da cerâmica $\mathrm{Al}_{2} \mathrm{O}_{3}-$ $4 \mathrm{wt}_{\mathrm{T}} \mathrm{Nb}_{2} \mathrm{O}_{5}$ e Kevlar ${ }^{\mathrm{TM}}$ obtidos em outros trabalhos [30]. Foi observado que mais de $50 \%$ se a dissipação de energia ocorreu na cerâmica, o que concorda com os resultados relatados anteriormente [32]. Em contrapartida, individualmente, os demais componentes de MAS dissipam menos de $7 \%$ da energia de bala de cada um. Em particular, o Kevlar ${ }^{\mathrm{TM}}$ dissipa uma quantidade relativamente baixa de energia $(0,22 \mathrm{~kJ})$ quando comparado com os compósitos de tecido de juta. O que corrobora com os resultados da Tabela 1, onde Kevlar ${ }^{\mathrm{TM}}$ possui desempenho balístico comparável aos compósitos de poliéster reforçados com tecido de juta em termos de profundidade de indentação. 
Tabela 3. Resultados de impacto e velocidade residual

\begin{tabular}{|c|c|c|c|c|}
\hline Componente do MAS & $V_{i}(m / s)$ & $\mathrm{V}_{\mathrm{r}}(\mathrm{m} / \mathrm{s})$ & $E(k J)$ & $\Delta E_{d}(\%)$ \\
\hline $\begin{array}{c}\qquad \mathrm{Al}_{2} \mathrm{O}_{3} \text { cerâmica } \\
\text { Poliéster com } 10 \% \text { vol tecido de juta } \\
\text { Poliéster com } 20 \% \text { vol tecido de juta } \\
\text { Poliéster com } 30 \% \text { vol tecido de juta } \\
\text { Kevlar }{ }^{\mathrm{TM}}\end{array}$ & $\begin{array}{l}848 \pm 6 \\
838 \pm 3 \\
837 \pm 4 \\
837 \pm 8 \\
848 \pm 6\end{array}$ & $\begin{array}{l}567 \pm 43 \\
805 \pm 7 \\
807 \pm 5 \\
812 \pm 8 \\
841 \pm 7\end{array}$ & $\begin{array}{l}1.60 \pm 0.300 \\
0.22 \pm 0.004 \\
0.24 \pm 0.006 \\
0.19 \pm 0.008 \\
0.22 \pm 0.001\end{array}$ & $\begin{array}{l}54.05 \\
6.22 \\
6.94 \\
5.37 \\
6.10\end{array}$ \\
\hline
\end{tabular}

A Tabela 4 apresenta uma análise econômica baseada no peso e no custo dos vários sistemas de blindagem multicamada. Os resultados nesta tabela são baseados em densidades medidas laboratoriais e preços fornecidos pelos fornecedores. Para fins de cálculo, a área de face da cerâmica foi considerada como 150x120 mm, semelhante aos outros componentes do MAS, e não o que foi realmente utilizado.

Tabela 4. Análise econômica e de peso dos materiais utilizados

\begin{tabular}{|c|c|c|c|c|c|}
\hline Componente da blindagem & $\begin{array}{l}\text { Volume } \\
\left(\mathrm{cm}^{3}\right)\end{array}$ & $\begin{array}{l}\text { Densidade } \\
\left(\mathrm{g} / \mathrm{cm}^{3}\right)\end{array}$ & Peso(kgf) & Preço por kg(U\$ dollars) & $\begin{array}{l}\text { Custo do } \\
\text { componente } \\
\text { (U\$ dollars) }\end{array}$ \\
\hline Cerâmica $\left(\mathrm{Al}_{2} \mathrm{O}_{3}+4 \% \mathrm{Nb}_{2} \mathrm{O}_{5}\right)$ & 190 & 3.72 & 0.707 & 2.18 & 1.54 \\
\hline Kevlar $^{\mathrm{TM}}$ & 190 & 1.44 & 0.274 & 63.60 & 17.43 \\
\hline Poliéster-10 vol\% tecido de juta & 190 & $1.12^{\mathrm{a}}$ & 0.213 & $3.90^{b}$ & 0.83 \\
\hline Poliéster-20 vol\% tecido de juta & 190 & $1.14^{\mathrm{a}}$ & 0.217 & $3.50^{b}$ & 0.76 \\
\hline Poliéster-30 vol\% tecido de juta & 190 & $1.16^{a}$ & 0.220 & $3.10^{b}$ & 0.68 \\
\hline Alumínio 5052-H34 & 95 & 2.68 & 0.255 & 5.10 & 1.30 \\
\hline Epóxi-30 vol\% tecido de juta & 190 & 1.13 & 0.215 & 8.38 & 1.80 \\
\hline \multicolumn{2}{|c|}{ Peso total com Kevlar ${ }^{\mathrm{TM}}$ (kgf) } & 1.236 & \multicolumn{2}{|c|}{ Custo total com Kevlar ${ }^{\mathrm{TM}}$} & 20.27 \\
\hline \multicolumn{2}{|c|}{$\begin{array}{c}\text { Peso total com Poliéster-10 vol\% tecido de } \\
\text { juta }\end{array}$} & 1.175 & \multicolumn{2}{|c|}{$\begin{array}{l}\text { Custo total com Poliéster-10 vol\% } \\
\text { tecido de juta }\end{array}$} & 3.67 \\
\hline \multicolumn{2}{|c|}{$\begin{array}{l}\text { Peso total com Poliéster-20 vol\% tecido de } \\
\text { juta }\end{array}$} & 1.179 & \multicolumn{2}{|c|}{$\begin{array}{c}\text { Custo total com Poliéster-20 vol\% } \\
\text { tecido de juta }\end{array}$} & 3.60 \\
\hline \multicolumn{2}{|c|}{$\begin{array}{l}\text { Peso total com Poliéster-30 vol\% tecido de } \\
\text { juta }\end{array}$} & 1.182 & \multicolumn{2}{|c|}{$\begin{array}{c}\text { Custo total com Poliéster-30 vol\% } \\
\text { tecido de juta }\end{array}$} & 3.53 \\
\hline \multicolumn{2}{|c|}{$\begin{array}{l}\text { Peso total com Epóxi-30 vol\% tecido de } \\
\text { juta }\end{array}$} & 1.177 & \multicolumn{2}{|c|}{$\begin{array}{c}\text { Custo total com Epóxi-30 vol\% } \\
\text { tecido de juta }\end{array}$} & 4.64 \\
\hline \multirow{2}{*}{\multicolumn{2}{|c|}{$\begin{array}{l}\text { Diminuição do peso (\%) do MAS com } \\
\text { compósito de tecido de juta em } \\
\text { comparação com Kevlar } \\
\text { Diminuição do peso (\%) do MAS com } \\
\text { compósito de tecido de juta em } \\
\text { comparação com Epóxi/Juta }\end{array}$}} & 5.400 & \multirow{2}{*}{\multicolumn{2}{|c|}{$\begin{array}{l}\text { Diminuição do custo (\%) do MAS } \\
\text { com compósito de tecido de juta } \\
\text { em comparação com Kevlar } \\
\text { Diminuição do custo (\%) do MAS } \\
\text { com compósito de tecido de juta } \\
\text { em comparação com Epóxi/Juta }\end{array}$}} & 474 \\
\hline & & 0.42 & & & 31.44 \\
\hline
\end{tabular}

a Tecido de juta: $1.3 \mathrm{~g} / \mathrm{cm}^{3}$; resina poliéster: $1.1 \mathrm{~g} / \mathrm{cm}^{3}$.

b Tecido de juta: US\$ 0.3; resina poliéster: US\$ 4.3.

Os resultados apresentados na Tabela 4 indicam que o uso de compósito de poliéster reforçado com tecido de juta de $30 \%$ em volume corresponde a cerca de $474 \%$ da redução de custo em comparação com o Kevlar $^{\mathrm{TM}}$ usado como camada secundária do MAS. Além disso, o mesmo compósito é $31,44 \%$ mais barato do que o similar equivalente a $30 \%$ em vol de epóxi reforçado com tecido de juta. Para a redução de peso, apenas uma porcentagem relativamente pequena, menor que $5 \%$, 
é obtida em MAS com $30 \%$ em volume, em comparação com MAS com Kevlar ${ }^{\text {TM }}$. Embora os compósitos Kevlar ${ }^{\mathrm{TM}}$ e de tecido de juta na Tabela 1 exibam praticamente o mesmo desempenho balístico, a economia de custos na Tabela 4 suporta a substituição do Kevlar ${ }^{\mathrm{TM}}$ por qualquer material compósito de juta. Além desta vantagem econômica, os compósitos de tecido de juta também estão associados a benefícios ambientais e sociais [33]. Hoje em dia, essas vantagens contribuem para uma indicação prática para as blindagens utilizarem compósito de poliéster reforçado com tecido de juta $30 \%$ em volume, pois a segunda camada do MAS é mais conveniente que Kevlar ${ }^{\mathrm{TM}}$ ou mesmo outros compósitos de tecido de juta.

\section{CONCLUSÃo}

- O sistema de blindagem multicamada compostos por uma cerâmica frontal, compósito de poliéster reforçado com tecido de juta, como segunda camada e uma placa de liga de alumínio traseira, atendem ao padrão balístico internacional.

- A profundidade de indentação do bloco de plastilina foi, estatisticamente, o mesmo para os compósitos de tecido de juta e para o Kevlar $^{\mathrm{TM}}$, quando utilizados com segunda camada em um MAS.

- Mecanismos de captura de fragmentos de cerâmica e bala são igualmente eficientes para compósitos de tecido Kevlar $^{\mathrm{TM}}$ e de juta. Isso também é verificado nos valores obtidos para impedância de onda de choque desses materiais.

- Apesar do desempenho balístico semelhante e da ligeira diferença de peso, o custo muito menor do compósito de poliéster reforçado com $30 \%$ vol de tecido de juta é menor do que o produzido com Kevlar ${ }^{\mathrm{TM}}$.

\section{Agradecimentos}

Os autores agradecem o apoio das agências brasileiras: CNPq, FAPERJ e CAPES para o desenvolvimento deste trabalho.

\section{REFERÊNCIAS}

1 NIJ Standard 0101.06, Ballistic Resistance of Personal Body Armor, US Depart. of Justice, July 2008.

2 Li R, Fan Q, Gao R, Huo L, Wang F, Wang Y. Effects of dynamic mechanical properties on the ballistic performance of new near- $\beta$ titanium alloy Ti684. Materials \& Design. 2014; 62:233-240. http://dx.doi.org/10.1016/j.matdes.2014.05.044

3 Tasdemirci A, Tunusoglu G and Guden M. The effect of the interlayer on the ballistic performance of ceramic/composite armors: experimental and numerical study. International Journal of Impact Engineering. 2012; 44:1-9. http://dx.doi. org/10.1016/j.jijimpeng.2011.12.005.

4 Jacobs MJN and Van Dingenen JLJ. Ballistic protection mechanisms in personal armor. Journal of Materials Science. 2001; 36(13):3137-3142. http://dx.doi.org/10.1023/A:1017922000090.

5 Abrate S. Ballistic impact on composite structures. 1st ed. Cambridge: Cambridge University Press; 1998. p. 215-220.

6 Lee YS, Wetzel ED and Wagner NJ. The ballistic impact characteristic of Kevlar® woven fabrics impregnated with a colloidal shear thickening fluid. Journal of Materials Science. 2003;

7 Hine PJ, Duckett RA., Morye SS., Carr DJ. and Ward IM.: Compos. Sci. Technol., 2000, vol. 60 , pp. 2631-42. 
8 Lee BL, Song JW, Ward JE. J. Compos. Mater., 1994, vol. 28, pp. 1202-26.

9 Medvedovski E. Ballistic performance of armor ceramics: influence of design and structure. Ceram. Int. 2010; 36: 2103-2127.

10 Meyers MA. Dynamic Behavior of Materials 1st ed. New York, USA: Wiley; 1994.183.

11 Louro LHL and Meyers MA. Effect of stress state and microstructural parameters on impact damage of alumina-based ceramics. Journal of Materials Science. 1989; 24(7):2516-2532. http://dx.doi.org/10.1007/BF01174523.

12 Mohanty AK, Misra M and Drzal LT. Sustainable bio-composites from renewable resources: opportunities and challenges in the green materials world. Journal of Polymers and the Environment. 2002; 10(1):19-26.

http://dx.doi.org/10.1023/A:1021013921916.

13 Netravali AN and Chabba S. Composites get greener. Materials Today. 2003; 6(4):2229. http://dx.doi.org/10.1016/S1369- 7021(03)00427-9.

14 Crocker J. Natural materials innovative natural composites. Materials Technology. 2008; 23(3):174-178. http://dx.doi. org/10.1179/175355508X373378.

15 John MJ and Thomas S. Biofibers and biocomposites. Carbohydrate Polymers. 2008; 71(3):343-364. http://dx.doi. org/10.1016/j.carbpol.2007.05.040.

16 Monteiro SN, Lopes FPD, Barbosa AP, Bevitori AB, Silva ILA and Costa LL. Natural lignocellulosic fibers as engineering materials: an overview. Metallurgical and Materials Transactions. A, Physical Metallurgy and Materials Science. 2011; 42(10):2963- 2974. http://dx.doi.org/10.1007/s11661-011-0789-6.

17 Faruk O, Bledzki AK, Fink HP and Sain M. Biocomposites reinforced with natural fibers. Progress in Polymer Science. 2012; 37(11):1555-1596. http://dx.doi.org/10.1016/j. progpolymsci.2012.04.003.

18 Thakur VK, Thakur MK and Gupta RK. Review: raw natural fibers based polymer composites. International Journal of Polymer Analysis and Characterization. 2014; 19(3):256-271. http://dx.doi.org/10.1080/1023666X.2014.880016.

19 Guven O, Monteiro SN, Moura EAB, Drelich W. Re-emerging field of Lignocellulosic fiber-polymer composites and ionizing radiation technology in then formulation. Polym Reviews 2016; 56: 702-736.

20 Holbery $\mathrm{J}$ and Houston D. Natural fiber reinforced polymer composites in automotive applications. JOM. 2006; 58(11):80- 86. http://dx.doi.org/10.1007/s11837-006-0234-2.

21 Zah R, Hischier R, Leal AL and Brown I. Curaua fibers in automobile industry: a sustainable assessment. Journal of Cleaner Production. 2007; 15(11-12):1032-1040. http://dx.doi. org/10.1016/j.jclepro.2006.05.036.

22 Zah R, Hischier R, Leal AL and Brown I. Curaua fibers in automobile industry: a sustainable assessment. Journal of Cleaner Production. 2007; 15(11-12):1032-1040. http://dx.doi. org/10.1016/j.jclepro.2006.05.036.

23 Ali A, Shaker ZR, Khalina A, Sapuan SM. Development of anti-ballistic board from ramie fiber. Polym Plast Technol Eng. 2011; 50: 622-634.

24 Wambua $P$, Vangrimde B, Lomov S, Verpoest I. The response of natural fibre composites to ballistic impact by fragment simulating projectiles. Compos. Struct. 2007; 77: 232-240.

25 Abidin MHZ, Mohamad MAH, Zaidi AMA, Mat WAW. Experimental study on ballistic resistance of sandwich panel protection structure with kenaf foam as a core material against small arm bullet. Appl. Mech. Mater. 2013; 315: 612-615.

26 da Cruz RB, Lima Jr. EP, Monteiro SN, Louro LHL. Giant Bamboo Fiber Reinforced Epoxy Composite in Multilayered Ballistic Armor. Mat. Res. 2015; 18: 70-75.

27 Monteiro SN, Louro LHL, Trindade W, Elias CN, Ferreira CL, Lima ES, Weber RP, Suarez JM, Figueiredo ABS, Pinheiro WA, da Silva LC, Lima Jr. EP. Natural curaua fiber-reinforced composites in multilayered ballistic armor. Metal. Mater. Trans. A. 2015; 46: 4567-4577.

28 Rohen LA, Margem FM, Monteiro SN, Vieira CMF, Araujo BM, Lima ES. Ballistic efficiency of an individual epoxy composite reinforced with sisal fibers in multilayered armor. Mater. Res. 2015; 18: 55-62. 
29 Monteiro SN, Braga FO, Lima Jr. EP, Louro LHL, Silva LC, Drelich JW. Promising curaua fiber-reinforced polyester composite. Polym. Eng. Sci. 2016; doi:10.1002/pen.24471.

30 Da Luz FS, Lima Jr EP, Louro LHL, Monteiro SN. Ballistic test of multilayered armor with intermediate epoxy composite reinforced with jute fabric. Mater. Res. 2015; 18: 170-177.

31 Monteiro SN, Milanezi TL, Louro LHL, Lima Jr. EP, Braga FO, Comes AV, Drelich JW. Novel ballistic ramie fabric composite competing with Kevlar ${ }^{\mathrm{TM}}$ fabric in multilayered armor. Mater. Desig. 2016; 96: 263-269.

32 Monteiro SN, Lima EP Jr, Louro LHL, Silva LC and Drelich JW. Unlocking function of aramid fibers in multilayered ballistic armor. Metallurgical and Materials Transactions A: Physical Metallurgy and Materials Science. 2015; 46(1):37-40. http://dx.doi.org/10.1007/s11661-014-2678-2.

33 Monteiro SN, Lopes FPD, Ferreira AS, Nascimento DCO. Natural fiber polymer matrix composites: cheaper, tougher and environmentally friendly. JOM. 2009; 61:17-22. 